This document is published in:

IEEE European Conference, EUROCON'13 (2013) pp. 391-398

DOI: 10.1109/EUROCON.2013.6625013

(C) 2013 IEEE. Personal use of this material is permitted. Permission from IEEE must be obtained for all other uses, in any current or future media, including reprinting/republishing this material for advertising or promotional purposes, creating new collective works, for resale or redistribution to servers or lists, or reuse of any copyrighted component of this work in other works. 


\title{
Comparison of Architectures for PAPR reduction in OFDM combining Pilot Symbols with Constellation Extension
}

\author{
Martha C. Paredes Paredes ${ }^{\# 1}$ and M. Julia Fernández-Getino García \#2 \\ \# Department of Signal Theory and Communications, Universidad Carlos III de Madrid \\ Avda. Universidad, 30, 28911 - Leganés, Madrid-Spain \\ ${ }^{1}$ mcparedes@tsc.uc3m.es \\ 2 mjuliadtsc.uc 3 m.es
}

\begin{abstract}
A main drawback of Orthogonal Frequency Division Multiplexing (OFDM) systems is that they suffer from a high Peak-to-Average Power Ratio (PAPR) at the transmitted signal. We propose three different architectures of a PAPR reduction technique combining pilot symbols with constellation extension. These architectures make use of a metric-based amplitude predistortion algorithm for the constellation extension embedded with orthogonal pilot symbols. Since neither the constellation extension nor the orthogonal pilots degrade the Bit Error Rate (BER), then the combined architectures also guarantee system performance. The three proposals outperform the previous algorithms (SAP and OPS) in terms of PAPR reduction, due to adequately joining pilots symbols with constellation extension. Moreover, the three architectures are examined from a complexity point of view, yielding a comparison in terms of computational load, what is straightforwardly related to implementation energy efficiency.

Index Terms-OFDM, peak power reduction, constellation extension, orthogonal pilot sequences
\end{abstract}

\section{INTRODUCTION}

The high Peak-to-Average Power Ratio (PAPR) is one of the most serious problems of the transmitted signal in Orthogonal Frequency Division Multiplexing (OFDM). The large peaks are critical because to avoid in-band distortion, which increases Bit Error Rate (BER), and out-of-band radiation, which causes adjacent channel interference, a High-PowerAmplifier (HPA) must be backed-off well below its maximum saturation output power, due to the infrequent peaks, which results in low efficiency.

There are several proposals to deal with the PAPR problem in OFDM systems [1], [2]. The simplest method is to deliberately clip the OFDM signal before amplification [3], [4]. Clipping can reduce PAPR but this is a nonlinear process and may cause in-band distortion and out-of-band interference. Then, there are techniques that cause no distortion and create no out-of-band radiation such as Partial Transmit Sequences (PTS) [5], Tone Reservation (TR) [6] and SeLected Mapping (SLM) [7]. However, these techniques may require the transmission of side information to the receiver, which reduces the transmission rate. Another type of PAPR reduction scheme is Active Constellation Extension (ACE) technique [8], which tries to alter the outer constellation points, that are moved within the proper quarter-plane such as the PAPR is minimized, while no BER degradation is induced. This scheme does not require special processing at reception. However, in ACE scheme there is an increase of the energy per symbol and its computational burden is high because of an iterative constellation extension process. The computational burden is reduced with a recent metric-based algorithm named Simple Amplitude Predistortion (SAP) [9], [10]. The metric of this method is defined to measure how much the input symbols contribute to the Inverse Discrete Fourier Transform (IDFT) output samples with large peaks. In SAP only a subset of symbols are amplitude predistorted (i.e. extended) with a constant scaling factor to avoid an exaggerated increase of the energy per symbol. On the other hand, Orthogonal Pilot Sequences (OPS) [11] is another approach, which inserts orthogonal pilot sequences in the input data to achieve PAPR reduction. Usually these pilot sequences are simultaneously employed in coherent OFDM channel estimation.

The idea of metric-based constellation extension embedded with pilot signaling was recently proposed in [12]. This means that SAP is aided by OPS method in order to outperform previous schemes. However, the combination of these methods lead to three different architectures of implementation, unlike the first architecture proposed in [12]. In this paper we compare these three different schemes in terms of PAPR reduction and complexity.

The rest of the paper is organized as follows. Section II briefly shows the OFDM signal model and PAPR definition problem. In Section III we present a review of SAP and OPS techniques. The three different architectures are proposed in Section IV. The analysis of complexity is exposed in Section V. In Section VI several simulation results are shown. Finally conclusions are drawn in Section VII.

\section{The OFDM SignAL MODEL}

The OFDM signal is the sum of $N$ independent signals modulated onto subchannels of equal bandwidth, which can be efficiently implemented by an IDFT operation. We denote $\mathbf{S}^{\ell}=\left\{S^{\ell}(k)\right\}_{k=0}^{N-1}$ as the input data sequence, consisting of the frequency-domain complex symbols over $k$ th subcarrier, $k=$ 
$\{0, \cdots, N-1\}$ to be transmitted in the $\ell$ th OFDM symbol. Then the time-domain signal $\mathbf{s}^{\ell}=\left\{s^{\ell}[n]\right\}_{n=0}^{N-1}$ is given by:

$$
s^{\ell}[n]=\frac{1}{\sqrt{N}} \sum_{k=0}^{N-1} S^{\ell}(k) e^{j \frac{2 \pi}{N} k n}, 0 \leq n<N-1
$$

where $k$ and $n$ are the frequency and time indices respectively.

The frequency-domain signal $\mathbf{S}^{\ell}=\left\{S^{\ell}(k)\right\}_{k=0}^{N-1}$ are independent, identically distributed (i.i.d.) random variables and due to the central limit theorem, a small percentage of output samples, will take very large magnitudes. This results in the well-known PAPR problem of OFDM systems. In general, the PAPR $(\chi)$ of the time-domain signal $\mathbf{s}^{\ell}=\left\{s^{\ell}[n]\right\}_{n=0}^{N-1}$ is mathematically defined as the ratio between the maximum instantaneous power and its average power [6], that is:

$$
\chi^{\ell}=\operatorname{PAPR}\left\{\mathbf{s}^{\ell}\right\}=\frac{\max \left(\left|\mathbf{s}^{\ell}\right|^{2}\right)}{E\left\{\left|\mathbf{s}^{\ell}\right|^{2}\right\}}
$$

where $E\{\cdot\}$ denotes expected value.

The most common way to evaluate the PAPR is to determine the probability that this PAPR exceeds a certain threshold $\chi_{0}$. This is represented by the Complementary Cumulative Distribution Function (CCDF) [13], which is a random variable, as:

$$
C C D F\left(\chi^{\ell}\right)=\operatorname{Prob}\left(\chi^{\ell}>\chi_{0}\right)=1-\left(1-e^{-\chi_{0}^{2}}\right)^{N}
$$

For convenience, we omit the use of superscript $\ell$ in the sequel.

\section{Conventional Algorithms}

As previously introduced, we describe here both techniques. In Sub-Section III-A, the metric-based constellation extension algorithm, denoted as Simple Amplitude Predistortion (SAP), is addressed. In Sub-Section III-B, the OPS technique is presented.

\section{A. Simple Amplitude Predistortion (SAP)}

The key of this method is to intelligently play with the outer constellation points such that the PAPR is minimized. In order to find the set of indices of the input data sequence that are moved within the proper quater-plane in the constellation, SAP employs a metric, defined mathematically in (4). This metric measures how much each frequency-domain symbol contributes to large peaks at the IDFT output. The frequencydomain symbols with the highest metric values are selected and predistorted with a constant scaling factor $\alpha$.

$$
\mu_{k}=\sum_{n \in S_{K}} \omega(n) f(n, k)
$$

where, $f(n, k)=-\cos \left(\varphi_{n k}\right)$ is a function which measures the phase angle $\left(\varphi_{n k}\right)$ between the output sample $s[n]$ and the contribution of symbol $S(k), \omega(n)=|\mathbf{s}|^{p}$ is a weighting function which gives more weight to the output samples with large magnitudes, $p$ is a selected parameter, and $S_{K}$ is a set of size $K$ whose elements are the indices of the output samples that are larger than a predetermined threshold value [9]. Then, the set of $\Gamma$ symbols (with cardinality $L=|\Gamma|$ ) with greatest positive metric are predistorted with a scaling factor $\alpha>1$.

The size of the subset $L$ and the value of the constant scaling factor $\alpha$ are chosen from a group of values suggested by the authors [9] and the alternatives are: $L=10$ with $\alpha=2$, $L=26$ with $\alpha=1.55$ and $L=40$ with $\alpha=1.3$.

\section{B. Orthogonal Pilot Sequences (OPS)}

In an OFDM symbol with $N$ subcarriers, a subset $\Upsilon$ of subcarriers will carry pilot symbols and thus, the input data sequence $\mathbf{S}$ is:

$$
S(k)= \begin{cases}X(k), & k \notin \Upsilon \\ P(k), & k \in \Upsilon\end{cases}
$$

where $X(k)$ and $P(k)$ are data and pilot symbols respectively.

The transmitted time-domain symbol $s[n]=x[n]+p[n]$ can be separated into two parts, as:

$$
s[n]=\left\{\begin{aligned}
x[n] & =\frac{1}{\sqrt{N}} \sum_{k \notin \Upsilon} X(k) e^{j \frac{2 \pi}{N} k n} \\
p[n] & =\frac{1}{\sqrt{N}} \sum_{k \in \Upsilon} P(k) e^{j \frac{2 \pi}{N} k n}
\end{aligned}\right.
$$

where $x[n]$ and $p[n]$ refer to the time-domain data and pilot signals, respectively.

OPS technique [11] proposes the use of a predetermined set of $M$ orthogonal pilot sequences of length $N_{p}\left(M \leq N_{p}\right)$ in order to reduce complexity and avoid any side information, since blind detection is possible at the receiver due to the orthogonality condition.

The $N_{p}$ pilot symbols of each OFDM symbol can be collected in a $N$-length sequence denoted as $\mathbf{P}$ where, the $k$ th element of this sequence is given by:

$$
[\mathbf{P}]_{k}= \begin{cases}P(k), & k \in \Upsilon \\ 0 & k \notin \Upsilon\end{cases}
$$

As stated before, a set of $M$ pilot sequences are available so the alphabet of $\mathbf{P}$ is $\left\{\mathbf{P}_{1}, \mathbf{P}_{2}, \cdots, \mathbf{P}_{M}\right\}$. Each pilot sequence of this finite set $\mathbf{P}_{m}, m \in\{1,2, \cdots, M\}$ contains the frequency-domain pilot symbols at pilot positions while zeros are inserted in the remaining ones. These pilot sequences are orthogonal so then the ortoghonality conditions is fulfilled:

$$
\left\langle\mathbf{P}_{m}, \mathbf{P}_{n}\right\rangle=0 \quad m \neq n \quad m, n=\{1, \cdots, M\}
$$

where $\langle\cdot, \cdot\rangle$ denotes the inner product.

In particular, if the well-known Walsh-Hadamard sequences are employed where $P(k) \in\{1,-1\}$, then $\left\langle\mathbf{P}_{m}, \mathbf{P}_{n}\right\rangle=$ $N_{p} \delta[m-n], m, n=\{1, \cdots, M\}$, where $\delta[\cdot]$ denotes the Kronecker delta function.

When implementing OPS, we can make use of time-domain processing to reduce complexity unlike OPS proposed in [11]. In this case, the orthogonal pilot sequences are previously generated in time-domain via IDFT-operation. They are stored and then used. Therefore, we avoid $(M-1)$ IDFT operations [12]. 


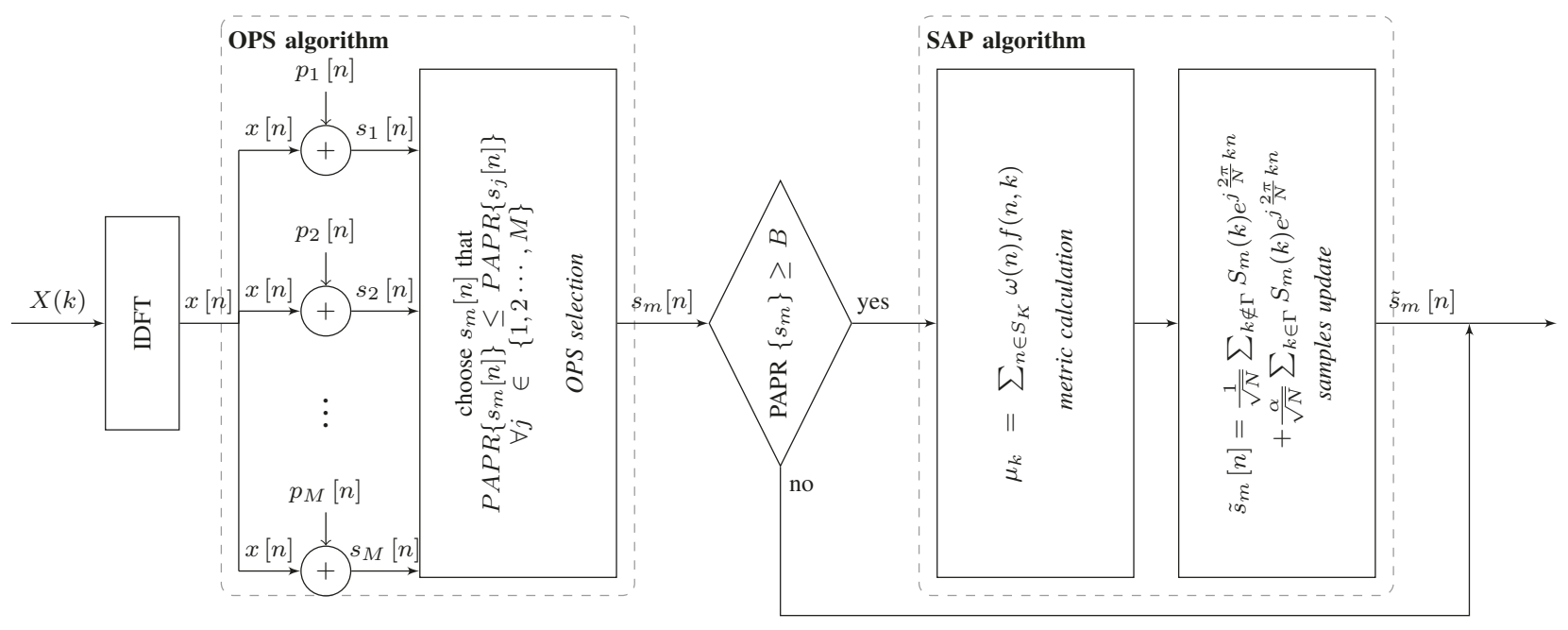

Fig. 1. Block diagram of architecture of scheme A.

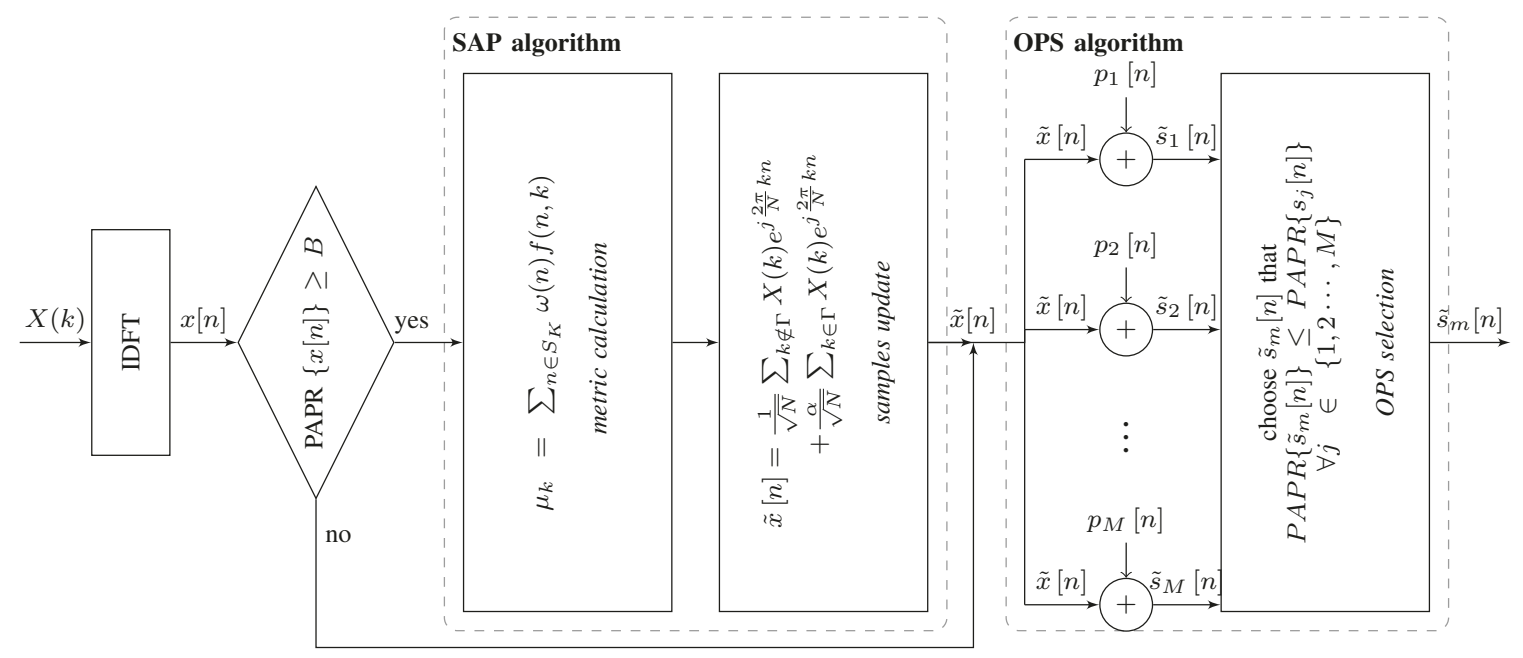

Fig. 2. Block diagram of architecture of scheme B.

\section{Proposed Architectures}

We consider to reduce the PAPR the use of SAP technique aided by OPS. The combination of two algorithms (SAP and OPS) presents three different architectures of implementation. Two of these schemes are two-step architectures according to the order of the algorithms and we refer to them as scheme A (OPS-SAP), already introduced in [12], and scheme B (SAP-OPS) respectively, and the third architecture, referred as scheme $\mathrm{C}$, is a simultaneous procedure. Each proposed architecture is explained next by using block diagrams in which the main building blocks are SAP and OPS stages.

The benefits of these architectures are that, first, they outperform previous methods (SAP, OPS) in terms of PAPR reduction. Also, from an efficiency point of view, we can carry out the constellation extension in these schemes with less energy per complex symbol than in SAP, if we adequately use the smart pilots to get an energy efficient system.

The drawbacks of this joint procedure are: (1) it is only intended for coherent systems with pilot symbols, and (2) it implies a slight increase in complexity, compared to SAP alone, since low-complex OPS must be carried out. However, this additional computational burden is negligible since only a search over $M$ sequences is performed.

\section{A. Scheme A: OPS-SAP}

The scheme A follows a two-step architecture that we show in Fig. 1, presented in [12]. OPS technique in time-domain is carried out at the first step, this is, the time-domain orthogonal pilot sequences $p_{m}[n], m \in\{1, \cdots, M\}$ are appropriately inserted, to obtain the lowest PAPR from the available set. Then, compute the PAPR and if this PAPR is larger than a predetermined threshold $B$ (this parameter can be taken as the value for which we obtain the greatest decrease on 


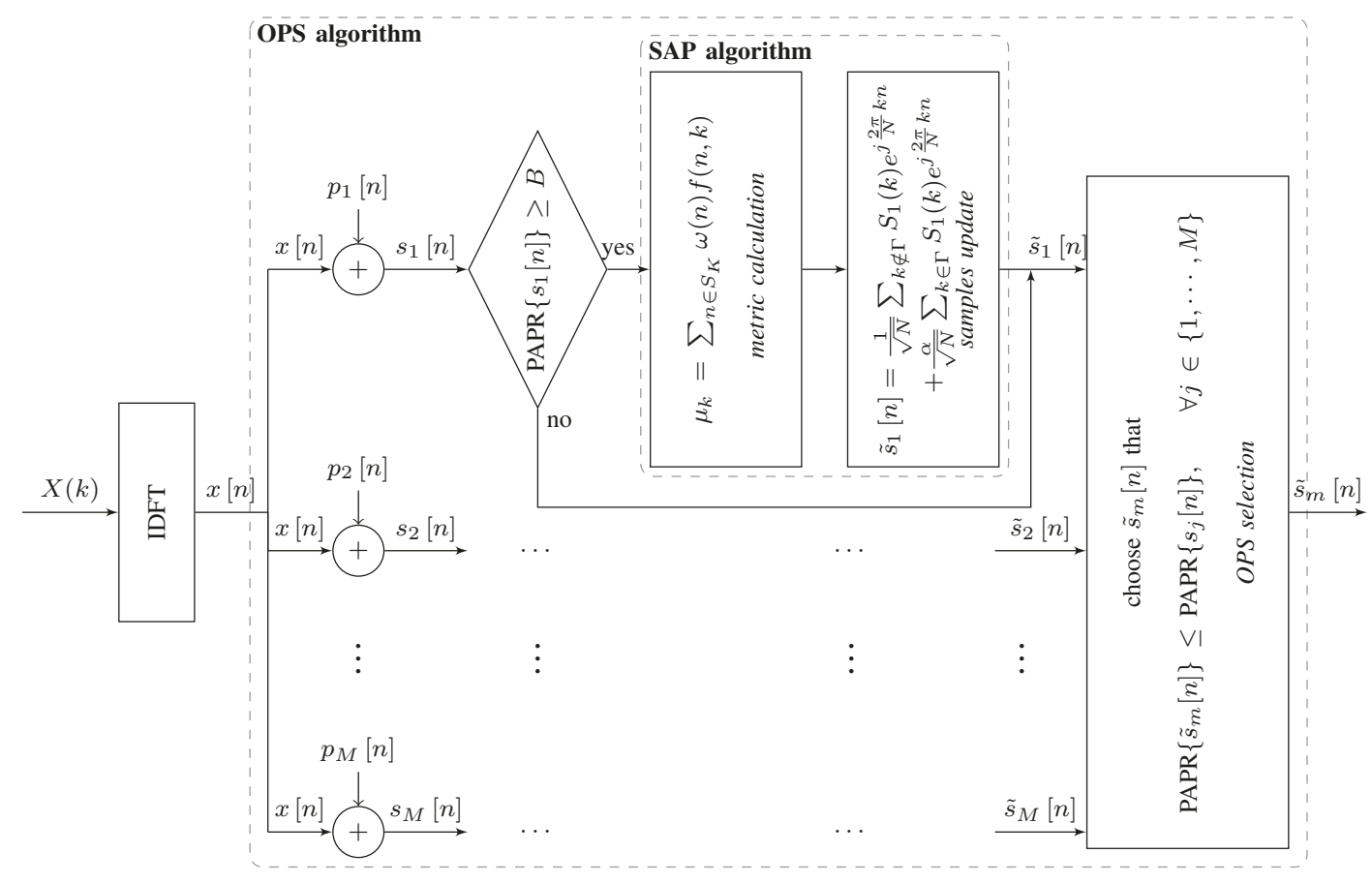

Fig. 3. Block diagram of architecture of scheme C.

average PAPR), we apply SAP algorithm. This second step consists in calculating the metric and then the frequencydomain symbols with greater positive metric are amplitude predistorted with a constant scaling factor $\alpha$. In this case, SAP algorithm is applied over both pilots and data symbols, allowing constellation extension of $N$ complex symbols.

\section{B. Scheme B: SAP-OPS}

The next two-step architecture is shown in Fig. 2. Once the IDFT operation of data input sequence $x[n]=\operatorname{IDFT}\{X(k)\}$ is fulfilled, the PAPR is calculated. This scheme B is applied whenever the PAPR of the data sequence is greater than a certain threshold $B$, and if this condition is true, SAP algorithm is executed at the first step. In this case SAP method is applied over $\left(N-N_{p}\right)$ complex symbols since only data symbols are employed. Then, we carry out OPS technique namely the timedomain orthogonal pilot sequences $p_{m}[n], m \in\{1, \cdots M\}$ are inserted in the constellation extended OFDM symbol. The OFDM symbol with the lower PAPR is selected to be transmitted.

\section{Scheme C: Simultaneous}

The architecture of scheme $\mathrm{C}$ is presented in Fig. 3 in which both SAP and OPS are carried out at the same time. In timedomain, the orthogonal pilots $p_{m}[n], m \in\{1, \cdots, M\}$ are inserted in their positions of the OFDM symbol. Then, for each $M$ branch of OPS scheme, the PAPR is computed and if this PAPR of the OFDM symbol is larger than threshold $B$, SAP algorithm is processed, which is applied over both pilot and data symbols, allowing constellation extension of
$N$ complex symbols. Once the $M$ branch are processed, the OFDM symbol with the lowest PAPR from the available set is selected to be transmitted. In this architecture, SAP algorithm is executed $(M-1)$ times more than in previous schemes.

\section{ANAlysis of COMPlEXity}

The total number of operations required to process any PAPR reduction technique is directly related to the complexity. In order to determine the best of the three architectures, in terms of complexity, we analyze the number of operations that each proposed scheme employs.

At the transmitter, in each proposed architecture the SAP and OPS schemes are executed. Therefore, we need to determine the total number of operations in each method. On one hand, the operations in OPS step are $M$ additions, $M N$-length maximum-value searches to find the PAPR in each sequence and a $M$-length minimum-index search to determine and select the pilot sequence with the lowest PAPR [12]. On the other hand, in SAP stage, the operations involve to calculate the metric that is proportional to $K N$ and the update in timedomain samples is proportional to $L N$ [9]. For Quadrature phase-shift keying (QPSK), the number of operations for the metric calculation are $7 K N$ real multiplications, $3 K N$ additions and $N$ divisions and the number of operations for the sample update are $5 L N$ real multiplications and $(2 L+1) N$ additions.

The number of operations to carry out the PAPR reduction process in each proposed scheme are presented in Table I. It is clearly observed that there are more operations in scheme $\mathrm{C}$, due to the SAP algorithm is executed in each $M$ branch of 
TABLE I

NUMBER OF OPERATIONS FOR EACH PROPOSED ARCHITECTURE

\begin{tabular}{cccc}
\hline Operations & Scheme A $(\boldsymbol{O P S}-\mathbf{S A P})$ & Scheme B $($ SAP-OPS) & Scheme C (Simultaneous) \\
\hline Real multiplications & $N(7 K+5 L)$ & $\left(N-N_{p}\right)(7 K+5 L)$ & $N M(7 K+5 L)$ \\
\hline Divisions & $N$ & $N-N p$ & $M N$ \\
\hline Additions & $M+N(3 K+2 L+1)$ & $M+\left(N-N_{p}\right)(3 K+2 L+1)$ & $M+N M(3 K+2 L+1)$ \\
\hline$M$-length min-index search & 1 & 1 & 1 \\
\hline$N$-length max-value search & $M$ & $M$ & $M$ \\
\hline
\end{tabular}

OPS, which means that the metric calculation and the sample time-domain update is carried out $M$ times while in scheme A and B the SAP stage is executed only once. Scheme B employs $\left(N-N_{p}\right)$ complex symbols in the first step because the pilot symbols have not been inserted yet, that is, the SAP procedure is performed over $\left(N-N_{p}\right)$ complex data symbols to calculate the metric and update time-domain samples, which involves a less consumption of resources than scheme A, that applies SAP over $N$ complex symbols.

\section{Simulation Results}

In this section, we present some simulations in order to analyze the performance and complexity (in terms of number of operations) of the proposed architectures. Computer simulations are evaluated by averaging over $10^{4}$ randomly generated OFDM symbols with QPSK modulation for $N=64$ and $N=256$ subcarriers. The threshold $B$ is fixed at $6 \mathrm{~dB}$. In OPS step, every $N_{f}$ subcarriers a pilot symbol is inserted, i.e. $N_{p}=N / N_{f}$ is the total number of pilots per OFDM symbol. We consider different values of $M$ to analyze the performance. On the other hand, the parameters of SAP step considered are $\{L=10$, with $\alpha=2\},\{L=26$, with $\alpha=1.55\}$ and $\{L=40$, with $\quad \alpha=1.3\}$ for all figures.

\section{A. Performance}

For comparison purposes, we present several figures with the same parameters for each proposed architecture. In these figures we illustrate the performance in terms of CCDF. These three schemes are evaluated for different values of parameters and also compared to OPS and SAP alone. In all figures, the solid-line curve corresponds to the conventional OFDM signal without any PAPR reduction technique, the dashed line curve represents OPS technique, the dash-dot marked line curves represent SAP algorithm and the dotted marked line curves correspond to the proposed schemes.

Scheme A (OPS-SAP) is illustrated in Fig. 4, 5 and 6. Fig. 4 represents an OFDM system for $N=64$ subcarriers with parameters $\left\{M=8, N_{p}=8\left(N_{f}=8\right)\right\}$, Fig. 5 for $N=256$ subcarriers with $\left\{M=8, N_{p}=8\left(N_{f}=32\right)\right\}$ and Fig. 6 for $N=256$ subcarriers with $\left\{M=N_{p}, N_{p}=32\left(N_{f}=8\right)\right\}$ in OPS stage, respectively. We observe in these figures that the improvement of proposed scheme (A) is in the order of 1.2 $\mathrm{dB}$ at a probability of $10^{-3}$ compared to SAP method.

Scheme $B$ (SAP-OPS) is shown in Fig. 7, 8 and 9. In Fig. 7 with $N=64$ subcarriers the parameters of OPS stage are $\left\{M=8, N_{p}=8\left(N_{f}=8\right)\right\}$. For Fig. 8 with $N=256$ subcarriers, in OPS stage the parameters are $\left\{M=8, N_{p}=\right.$

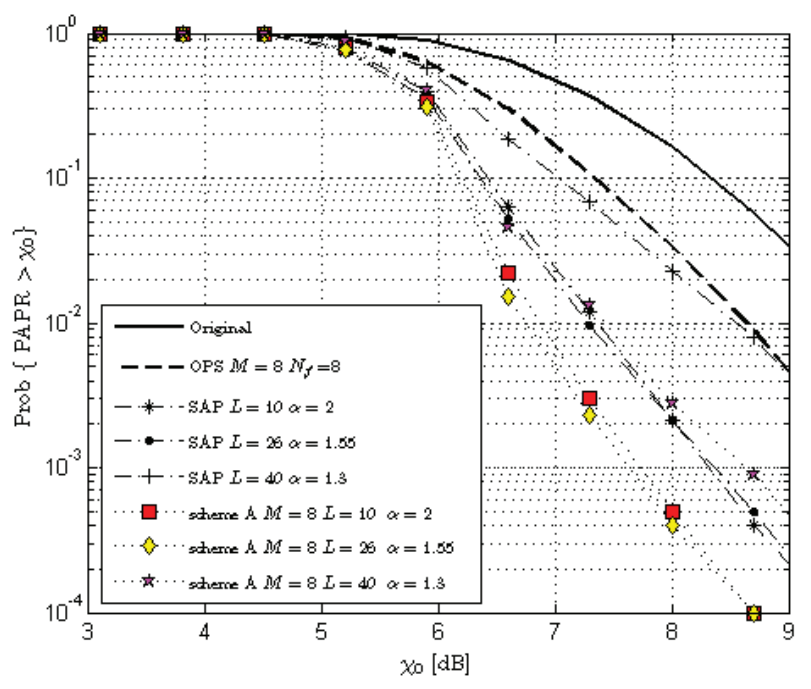

Fig. 4. CCDF of the PAPR for $N=64$ with $M=8$ and $N_{p}=8(N f=8)$. Scheme A with the same parameters than OPS and SAP.

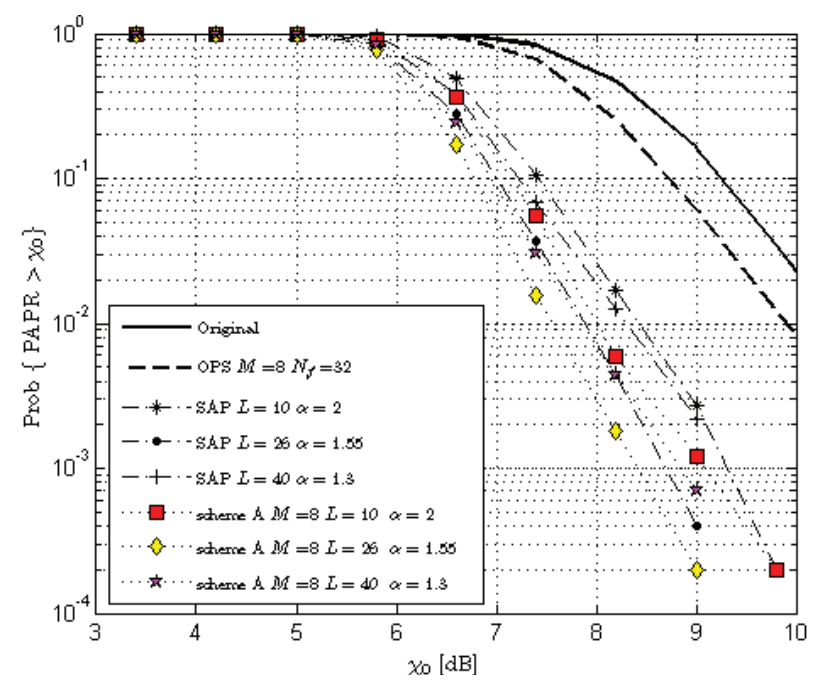

Fig. 5. CCDF of the PAPR for $N=256$ with $M=8$ and $N_{p}=8$ $(N f=32)$. Scheme A with the same parameters than OPS and SAP.

$\left.8\left(N_{f}=32\right)\right\}$. In Fig. 9 with $N=256$ subcarriers the parameters in OPS stage are $\left\{M=N_{p}, N_{p}=32\left(N_{f}=8\right)\right\}$. We observe in these figures that the improvement of this scheme $\mathrm{B}$ is in the order of $0.8 \mathrm{~dB}$ at a probability of $10^{-3}$ compared to SAP algorithm. 


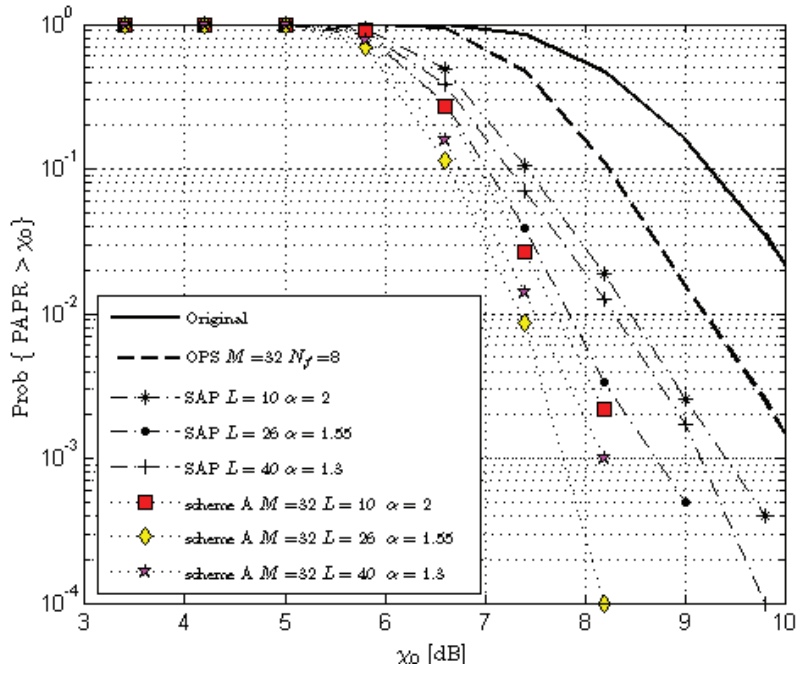

Fig. 6. CCDF of the PAPR for $N=256$ with $M=N_{p}$ and $N_{p}=32$ $(N f=8)$. Scheme A with the same parameters than OPS and SAP.

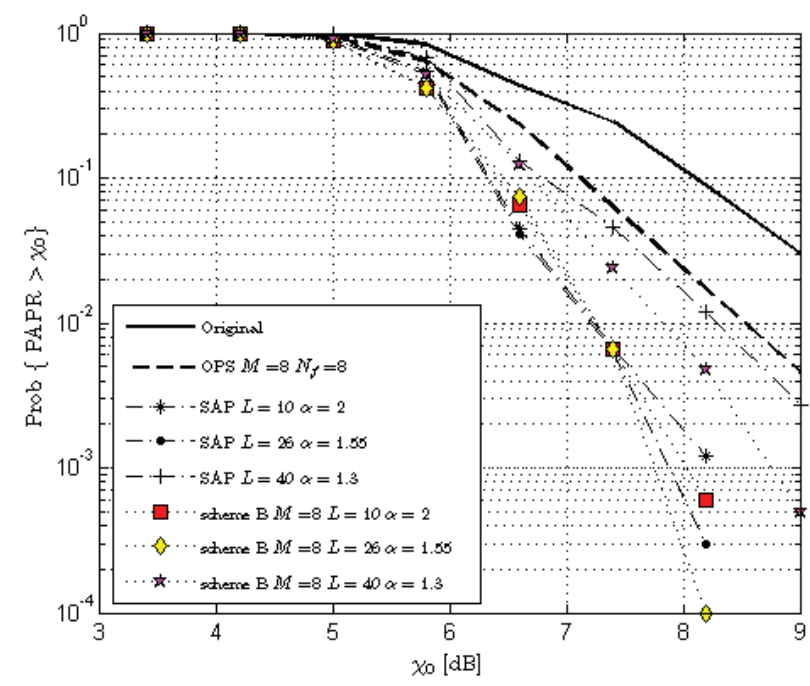

Fig. 7. $\mathrm{CCDF}$ of the PAPR for $N=64$. OPS with $M=8$ and $N_{p}=8$ $(N f=8)$. Scheme B with the same parameters than SAP and OPS.

Scheme $C$ (Simultaneous) is depicted in Fig. 10, 11 and 12 for $N=64$ and $N=256$ subcarriers. Fig. 10 with $N=$ 64 subcarriers, in OPS step the parameters that are used are $\left\{M=8, N_{p}=8\left(N_{f}=8\right)\right\}$. Fig. 11 for $N=256$ with $\left\{M=8, N_{p}=8\left(N_{f}=32\right)\right\}$. Fig. 12 for $N=256$ with $\left\{M=N_{p}, N_{p}=32\left(N_{f}=8\right)\right\}$. We observe in these figures that the improvement of proposed scheme (C) is in the order of $1.3 \mathrm{~dB}$ at probability of $10^{-3}$.

For comparison purposes in terms of performance, the schemes A, B and C are presented in Fig. 13 and 14 for $N=64$ and $N=256$ subcarriers respectively. These curves indicate that the performance of scheme $\mathrm{C}$ is slightly better compared to other ones.

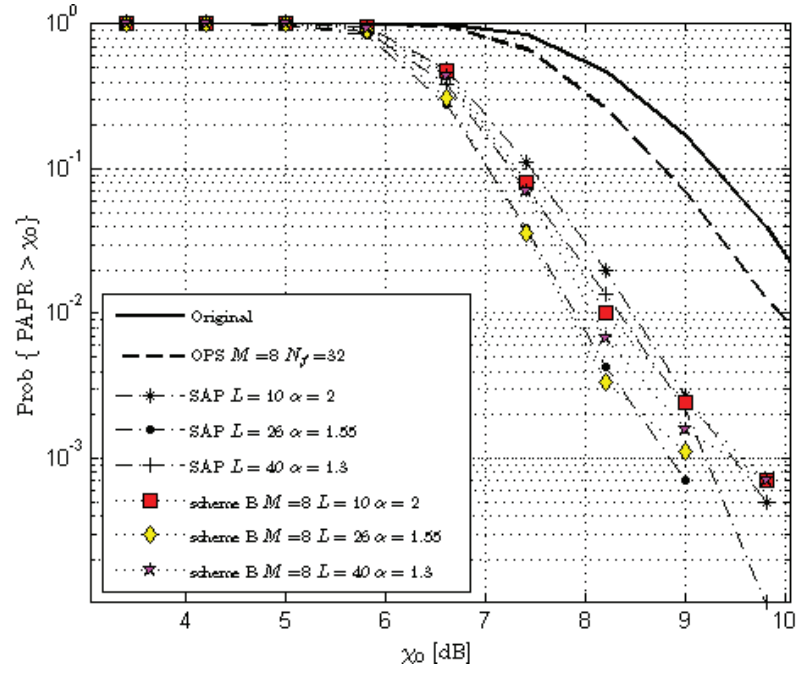

Fig. 8. CCDF for $N=256$. OPS with $M=8$ and $N_{p}=8(N f=32)$. Scheme B with the same parameters than SAP and OPS.

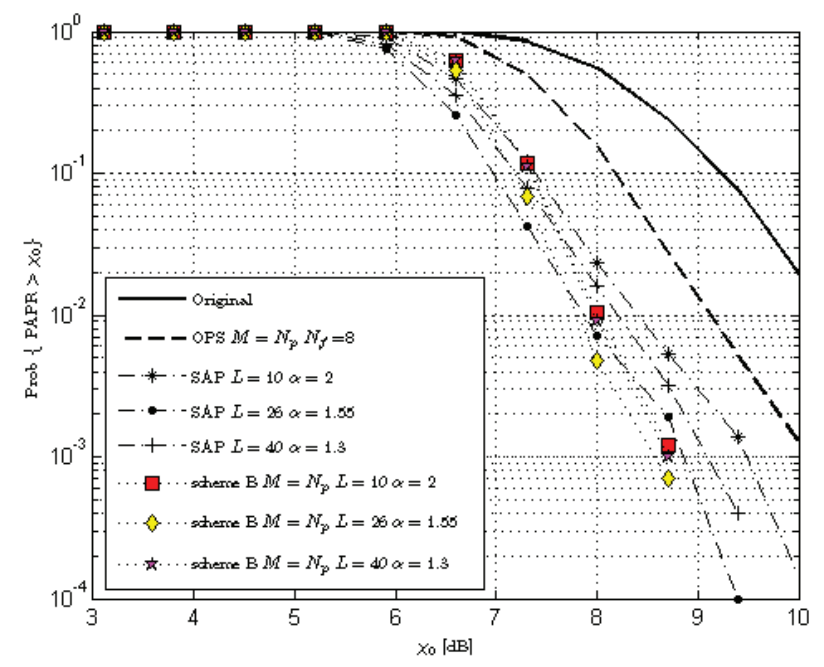

Fig. 9. CCDF for $N=256$. OPS with $M=N p$ and $N_{p}=32(N f=8)$. Scheme B with the same parameters than SAP and OPS.

\section{B. Complexity}

The number of operations that schemes A, B and C employ are shown in Table II and III for $N=64$ and $N=256$ subcarriers, respectively. For the OFDM system with $N=64$ subcarriers (Table II), the different parameters for SAP and OPS stages are $M=8$ with $N_{p}=8\left(N_{f}=8\right)$ and $\{\alpha=$ $2, L=10\}$. For the OFDM system with $N=256$ subcarriers (Table III) the parameters are $M=8$ with $N_{p}=8\left(N_{f}=32\right)$ and $\{\alpha=2, L=10\}$. From the comparison in Table II and III we can observe that scheme B (SAP-OPS) is the best in terms of complexity compared to other ones since it employs a lower number of operations. 


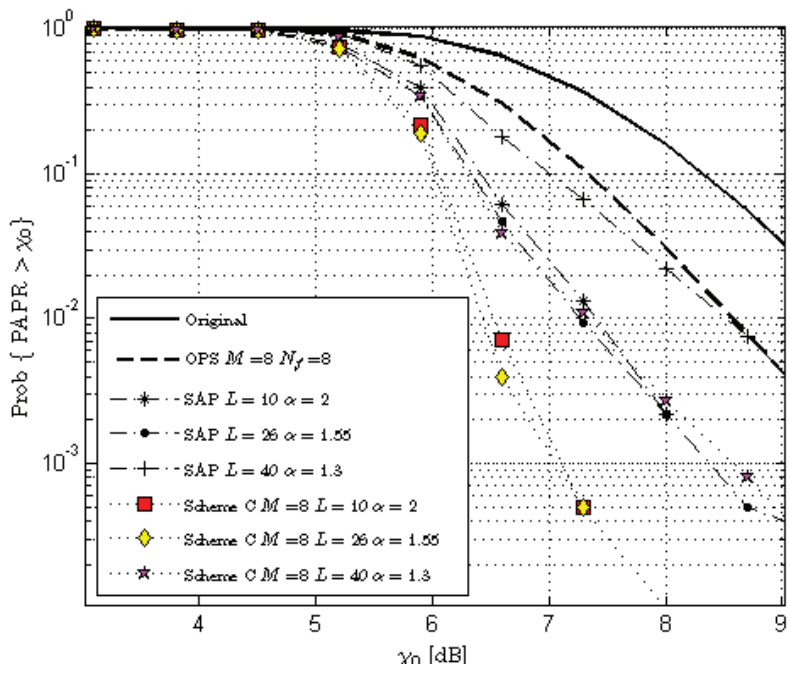

Fig. 10. CCDF of the PAPR for $N=64$ subcarriers. OPS with $M=8$ and $N_{p}=8(N f=8)$. Scheme $\mathrm{C}$ with the same parameters than SAP and OPS.

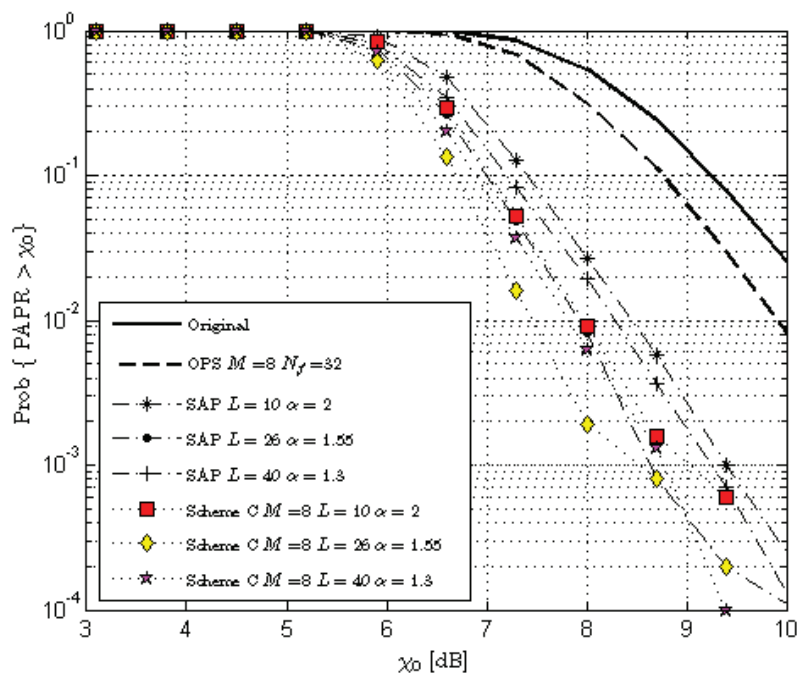

Fig. 11. CCDF of the PAPR for $N=256$ subcarriers. OPS with $M=8$ and $N_{p}=8(N f=32)$. Scheme C with the same parameters than SAP and OPS.

\section{CONCLUSION}

One of the most serious problems in an OFDM system is the high Peak-to-Average Power Ratio (PAPR) of the transmitted signal. In this paper, we combat the PAPR problem of OFDM systems with the combination of pilot symbols with constellation extension. The combination of these techniques can present three different architectures of implementation, which depends on the position of the algorithms. Two of the schemes are two-step architectures and the third one is a simultaneous scheme. The main aim of our work is to propose the three new architectures and determine the most adequate to get a good performance with a lower complexity. We found that the scheme B (SAP-OPS) employs a lower number of

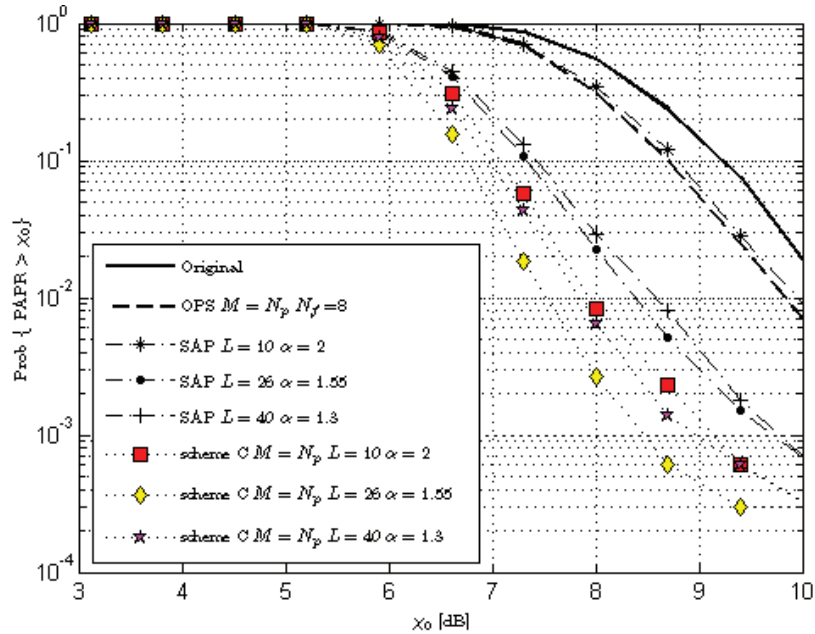

Fig. 12. CCDF of the PAPR for $N=256$ subcarriers. OPS with $M=N_{p}$ and $N_{p}=32(N f=8)$. Scheme $\mathrm{C}$ with the same parameters than SAP and OPS

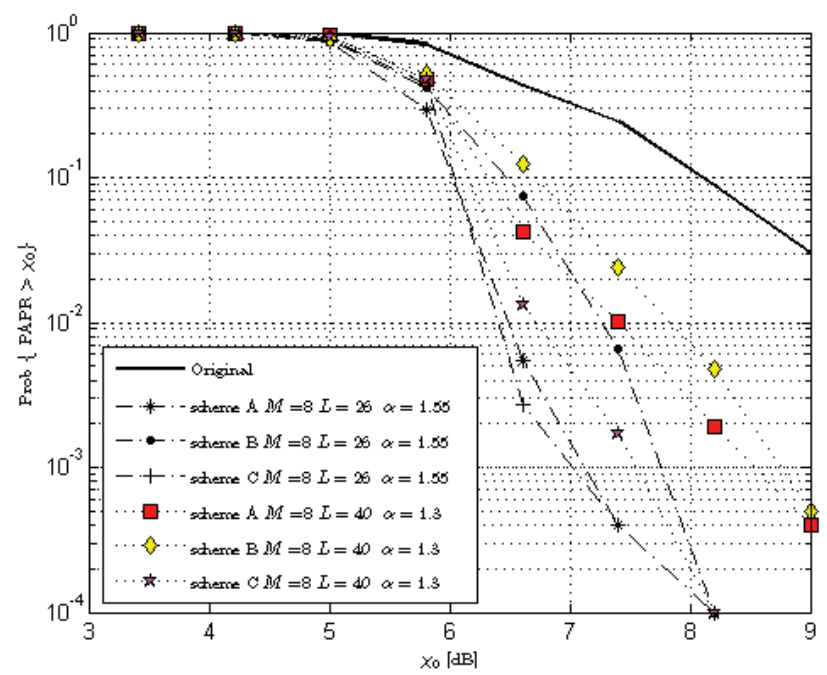

Fig. 13. CCDF of the PAPR for $N=64$, with parameter $M=8$ and $N_{p}=$ $8(N f=8)$ in OPS step and $\{\alpha=1.55, L=26\}$ and $\{\alpha=1.3, L=40\}$ in SAP stage.

operations to carry out the PAPR reduction process while the scheme $\mathrm{C}$ is slightly better in terms of performance compared to other ones.

\section{ACKNOWLEDGMENT}

This work has been partly funded by the Spanish national projects GRE3N-SYST (TEC2011-29006-C03-03) and COMONSENS (CSD2008-00010), and SENESCYT (Ecuador).

\section{REFERENCES}

[1] T. Jiang and Y. Wu, "An overview: Peak-to-average power ratio reduction techniques for OFDM signals”, IEEE Trans. on Broadcasting, vol. 54, no. 2, pp. 257-268, Jun. 2008. 


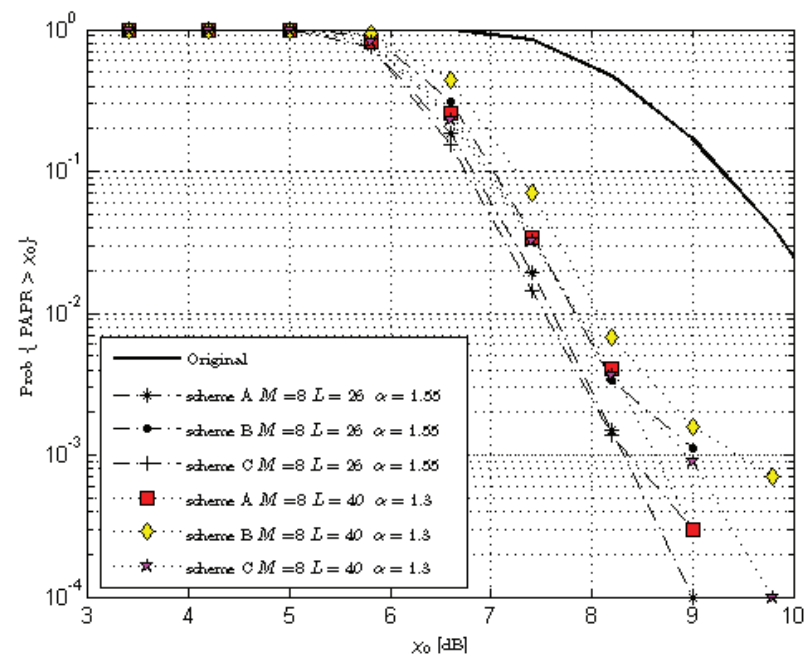

Fig. 14. CCDF of the PAPR for $N=256$ with parameter $M=8$ and $N_{p}=8(N f=32)$ in OPS stage and $\{\alpha=1.55, L=26\}$ and $\{\alpha=$ $1.3, L=40\}$ in SAP stage.

TABLE II

Number of Operations FOR EACH PROPOSED ARCHITECTURE WITH $N=64$ SUBCARRIERS

\begin{tabular}{cccc}
\hline Operations & Scheme $\boldsymbol{A}$ & Scheme $\boldsymbol{B}$ & Scheme $\boldsymbol{C}$ \\
\hline Real multiplications & 20403 & 17853 & 163230 \\
\hline Divisions & 64 & 56 & 520 \\
\hline Additions & 87248 & 76352 & 69742 \\
\hline$M$-length min-index search & 1 & 1 & 1 \\
\hline$N$-length max-value search & 8 & 8 & 8 \\
\hline
\end{tabular}

[2] Seung Hee Han and Jae Hong Lee, "An overview of peak-to-average power ratio reduction techniques for multicarrier transmission”, IEEE Wireless Communications, vol. 12, no. 2, pp. 56-65, April 2005.

[3] H. Ochiai and H. Imai, "Performance analysis of deliberately clipped OFDM signals", IEEE Trans. on Communications, vol. 50, no. 1, pp. 89-101, Jan. 2002.

[4] J. Armstrong, "Peak-to-Average power reduction for OFDM by repeated clipping and frequency domain filtering”, Electronics Letters, vol. 38, no. 8, pp. 246-247, Feb. 2002.

[5] S. H. Muller and J. B. Huber, "OFDM with reduced peak-to-average power ratio by optimum combination of partial transmit sequences", Electronics Letters, vol. 33, no. 5, pp. 368-369, 1997.

[6] J. Tellado, "Multicarrier modulation with low PAR: applications to DSL and wireless", Kluwer Academic Publishers, 2002.

[7] R. W. Bauml, R. F. H. Fisher and J. B. Huber, "Reducing the Peak-toAverage Power Ratio of Multicarrier Modulation by Selected Mapping", Electronics Letters, vol. 32, no. 22, pp. 20562057, Oct. 1996.

[8] B. S. Krongold and D. L. Jones, "PAR Reduction in OFDM via Active Constellation Extension”, IEEE Trans. on Broadcasting, vol. 49, no. 3, pp. 258-268, Sept. 2003.

[9] S. Sezginer, and H. Sari, "OFDM Peak Power Reduction with Simple Amplitude Predistortion”, IEEE Communications Letters, vol. 10, no. 12, pp. 65-67, Feb. 2006.

[10] S. Sezginer, and H. Sari, "Metric-Based Symbol Predistortion Techniques for Peak Power Reduction in OFDM Systems", IEEE Trans. on Wireless Communications, vol. 6, no. 7, pp. 2622-2629, Jul. 2007.

[11] M. J. Fernandez-Getino Garcia, O. Edfors, and J. M. Paez-Borrallo, "Peak power reduction for OFDM systems with orthogonal pilot sequences", IEEE Trans. on Wireless Communications, vol. 5, no. 1, pp. 47-51, Jan. 2006.

[12] M. C. Paredes, and M. J. Fernández-Getino García, "Energy Efficient Peak Power Reduction in OFDM with Amplitude Predistortion aided by
TABLE III

Number of Operations For EACH Proposed ARCHitecture With $N=256$ SUBCARRIERS

\begin{tabular}{cccc}
\hline Operations & Scheme A & Scheme B & Scheme C \\
\hline Real multiplications & 371200 & 359600 & 2969600 \\
\hline Divisions & 256 & 248 & 2048 \\
\hline Additions & 158984 & 154016 & 1271816 \\
\hline$M$-length min-index search & 1 & 1 & 1 \\
\hline$N$-length max-value search & 8 & 8 & 8 \\
\hline
\end{tabular}

Orthogonal Pilots", IEEE Trans. on Consumer Electronics vol. 59, no. 1, pp. 45-53, Feb. 2013.

[13] H. Ochai, and H. Imai, "On the Distribution of the Peak-to-Average Power Ratio in OFDM signal”, IEEE Trans. on Communications, vol. 49, no. 23, pp. 282-289, Feb. 2001. 